\title{
Identification of Wave Energy Device Models From Numerical Wave Tank Data_Part 1: Numerical Wave Tank Identification Tests
}

\author{
Josh Davidson, Simone Giorgi, and John V. Ringwood, Senior Member, IEEE
}

\begin{abstract}
In this paper and its companion [1], the identification of mathematical models describing the behaviour of wave energy devices (WECs) in the ocean is investigated through the use of numerical wave tank (NWT) experiments. This paper deals with the identification tests used to produce the data for the model identification. NWTs, implemented using computational fluid dynamics (CFD), are shown as an effective platform to perform the identification tests. The design of the NWT experiments, to ensure the production of information-rich data for the model identification, is discussed. A case study is presented to illustrate the design and implementation of NWT experiments for the identification of WEC models.
\end{abstract}

Index Terms-Wave energy conversion, hydrodynamic modelling, system identification, numerical wave tank, computational fluid dynamics.

\section{INTRODUCTION}

H YDRODYNAMIC models are important for the design, simulation and control of wave energy converters (WECs). In the wave energy community, the hydrodynamic models are normally formulated under the assumptions of small body motions and wave heights, in an incompressible, inviscid and irrotational fluid of constant density. Fully describing the dynamics of the fluid and its interaction with a structure involves solving the Navier-Stokes equations, which historically have been simplified to obtain a linear potential flow equivalent, whereby solutions are generated by linearising the problem through assumptions of small amplitude oscillations. This is a major limitation of this modelling approach, since WECS are designed to operate over a wide range of sea conditions where large amplitude motions will result from energetic waves or sustained wave/WEC resonance. At this expanded amplitude range, the linearising assumptions are invalid, as nonlinear effects become relevant.

An alternative modelling approach, detailed in this paper and its companion, is that of system identification, where models are determined from input/output data measured from the system under study [2]. System identification, which can be

Manuscript received July 31, 2015; revised November 26, 2015; accepted January 01, 2016. This work was supported in part by the Enterprise Ireland under Grant EI/CF/2011/1320, in part by the Irish Government and the European Union under Ireland's EU Structural and Investment Funds Programme 2014-2020, and in part by the Science Foundation Ireland under Grant 13/IA/1886. Paper no. TSTE-00634-2015.

The authors are with the Centre for Ocean Energy Research, Maynooth University, Maynooth, Ireland (e-mail: john.ringwood@eeng.nuim.ie).

Color versions of one or more of the figures in this paper are available online at http://ieeexplore.iee.org.

Digital Object Identifier 10.1109/TSTE.2016.2515512 considered to be the dynamical equivalent of static function approximation, has its origins in the methods developed by Legendre, Laplace and Gauss in the 19th century, which were used to fit functional forms to (typically) astronomical data [3]. However, since the 1960's, the field of dynamical system identification has been consolidated and many successful applications have been reported using a wide variety of methods across a broad range of application areas [4].

The fundamental principle of system identification is captured in Fig.1. The identification procedure follows the following steps:

1) A parametric structure is chosen for the model,

2) A suitable input signal, $u$ is synthesised and input to the system,

3) The input signal, $u$ and resulting output signal, $y$ are recorded,

4) An identification algorithm is used to determine the optimal parameter vector, $\Theta$, which minimises some error metric between the actual measured output $y$ and that produced by the identified parametric model.

One major difficulty in system identification is ensuring that the input/output data used to determine the model is sufficiently representative of the system dynamics and, in particular, must cover the range of frequencies and amplitudes likely to be encountered during system operation. In the WEC case, such a range of excitation signals are not likely to be available in the open ocean (at least not in a reasonably short time frame) and there are difficulties in exactly enumerating the excitation experienced by the device, particularly for a directional device. In short, there is no external control of the excitation. Alternatively, it is possible to employ tank tests. However, in addition to the significant cost and the need for a physical prototype, there may be limitations on the range of excitation signals available and tank wall reflections may further limit the range and duration of viable tests. One other possibility for generating suitable input/output data is to use a numerical wave tank (NWT) implemented with computational fluid dynamics (CFD).

The use of CFD-based NWTs to identify hydrodynamic parameters has been reported by a number of researchers. For example, [5] used CFD experiments to identify a coefficient for a viscous damping term to be added to the Cummins equation. [6] performed harmonic oscillation experiments to determine the added mass and radiation resistance coefficients of a floating body at discrete frequencies. [7] and [8] used free decay experiments to identify the full state dynamics of 


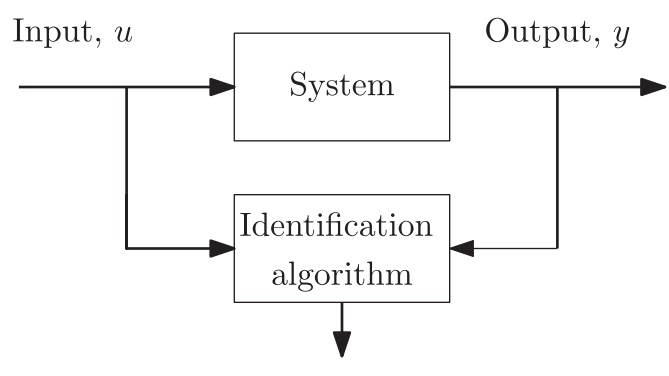

Parameters, $\Theta$

Fig. 1. System identification principle.

a floating body. [9] used free decay experiments and input waves to identify the state plus input dynamics of an oscillating water column (OWC). [10] identified the state plus input dynamics of a floating body, by introducing a power take-off (PTO) force to the body to drive its motion and also determined nonlinear hydrodynamic restoring force parameters from the NWT experiments. [11] used input waves to identify nonlinear hydrodynamic excitation force kernals. [12] gives an initial exposition of NWT experiments for the identification of hydrodynamic models and shows examples of both input waves and PTO forces in NWT experiments to train and validate a generalised hydrodynamic model. The work reported in [12] is a precursor to the more comprehensive study documented in this paper and in [1].

This paper focusses on the NWT experiments designed to provide information-rich data for the identification of the hydrodynamic models detailed in [1]. Section II gives an overview of NWTs and the underlying CFD used to implement the NWT simulations. The design of the NWT experiments is discussed in Section III. A case study illustrating the methods described in this paper is presented in Section IV and discussed in Section V. Conclusions are drawn in Section VI.

\section{NUMERICAL WAVE TANKS}

A NWT is the generic name of numerical simulators for modelling nonlinear free surface waves, hydrodynamic forces and floating body motions [13]. Current day computing power allows the implementation of NWTs using CFD.

\section{A. Computational Fluid Dynamics}

The dynamics of fluids is governed by the transfer of mass, momentum and heat. These three processes are described by the Navier-Stokes equations, a set of partial differential equations derived in the early nineteenth century, which form the basis for the analysis of fluid dynamics [14].

Continuity equation:

$$
\frac{\partial \rho}{\partial t}+\nabla \cdot(\rho \mathbf{u})=0
$$

Momentum equation:

$$
\rho \frac{\partial \mathbf{u}}{\partial t}=\nabla \cdot \tau_{i j}
$$

Energy equation:

$$
\rho \frac{\partial e}{\partial t}=\nabla \cdot(k \nabla T)-p \nabla \cdot \mathbf{u}+\tau_{i j}^{v} \frac{\delta u_{i}}{\delta x_{j}} .
$$

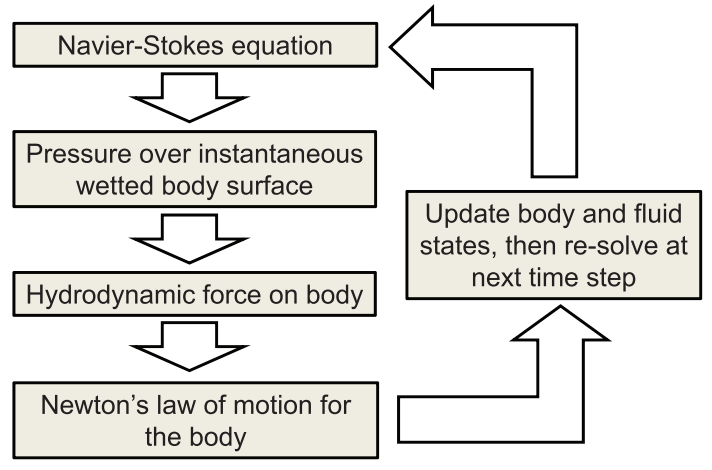

Fig. 2. Schematic of CFD process for fluid-structure interaction.

where $\rho$ is the fluid density, $\mathbf{u}$ the velocity, $e$ the internal energy, $T$ the temperature, $k$ the thermal conductivity and $\tau_{i j}$ is the stress tensor comprising the pressure, $-p \delta_{i j}$, and viscous terms, $\tau_{i j}^{v}$ :

$$
\tau_{i j}^{v}=\mu\left\{\frac{\delta u_{i}}{\delta x_{j}}+\frac{\delta u_{j}}{\delta x_{i}}\right\}+\delta_{i j} \lambda \nabla \cdot \mathbf{u} .
$$

where $\mu$ is the coefficient of viscosity, $\delta_{i j}$ is the Kronecker delta function and $\lambda$ is the bulk viscosity.

The coupled continuity, momentum and energy equations, Eqs. (1)-(3), are indeterminate and require two more equations to obtain closure which are provided by the ideal gas laws:

$$
p=\rho R T \text {. }
$$

and

$$
e=c_{v} T
$$

where $c_{v}$ is the specific heat at constant volume.

In general, these equations have no known analytical solution, however, they may be solved numerically using CFD by discretising the domains of space and time to form a system of linear algebraic equations, which are computer implementable. CFD treats the fluid-structure interaction problem, using the scheme outlined in Fig. 2. This process allows fully nonlinear hydrodynamic calculations, including effects neglected by traditional linear velocity potential methods such as viscosity, large wave amplitudes and body motions, green water and vortex shedding, however at great computational expense.

The use of CFD to simulate wave energy converters has been validated against experimental data and shown to produce accurate results by many researchers, see for example [15], [16], [17] and [18]. The focus of this paper is not on the validation of the CFD simulations, but rather on their use for the identification of hydrodynamic models, and therefore assumes that the CFD simulations are sufficiently accurate.

\section{B. NWT Experiments for Identification of Hydrodynamic Models}

Using a CFD based NWT to generate data for WEC model identification has the following advantages:

- Reflections from 'tank' walls can be effectively controlled by numerical absorption techniques,

- Can test the device at full scale, eliminating scaling effects, 
- A wide variety of excitation signals which are useful for system identification can be implemented, including incident waves, forces directly applied to the device and free response tests,

- The device can be constrained to different modes of motion without requiring mechanical restraints, which can add friction and alter the device dynamics,

- Hydrodynamic force on the body can be measured,

- Signals can be passively measured without requiring physical sensor devices, which can alter the device or fluid dynamics and are subject to measurement error, and, most importantly,

- Specialist equipment, including a prototype WEC device, is not required.

However, NWTs are not without their drawbacks. The chief disadvantage, over a conventional wave tank, is the excessively long time to perform the numerical computation of the response, and typical computation times can be up to 1000 times the simulation time i.e. $1 \mathrm{~s}$ of simulation time takes $1000 \mathrm{~s}$ to compute. The modelling method presented in this paper and [1] directly addresses the computational complexity issue, by using NWTs to develop sets of representative responses, which can subsequently be used to produce computationally efficient parametric models. The construction of these system identification tests needs to be carefully considered, so that representative input/output behaviour over the operational space is recorded, while minimising overall computation time.

\section{EXPERIMENT DESIGN}

One important issue in system identification is the specification of the system input signal. The signal should excite the system over its whole range of operation, providing information of the system's behaviour for all the conditions which the identified model will be required to replicate. For example, a purely sinusoidal input signal will only give information of the system's behaviour at that single frequency. In general, a good test signal should satisfy the following properties:

- Good coverage of the frequencies where the system has a significant non-zero frequency response,

- Good coverage of the full input and output signal ranges (if the system is nonlinear), and

- Economic use of the test time.

Typically useful signals for the identification of linear systems are pseudo-random binary sequences, which have a flat frequency spectrum, or multi-sinusoids which contain a set of closely-packed frequencies [19]. For nonlinear systems, there is an additional input/output signal amplitude dependence in the model, so the full operational range of amplitudes will also, ideally, need to be present in the identification signal. Therefore pseudo-random sequences with randomly varying amplitudes (or random amplitude, random period [RARP]) can be employed for nonlinear systems, while chirp and multi-sine signals are also useful, since the input signal varies continuously over the allowable input amplitude range.

Fig. 3 shows an examples of chirp (a), RARP (b) and multisine (c) signals. The spectral content and amplitude distributions of these signals are then plotted in Fig. 4, displaying their

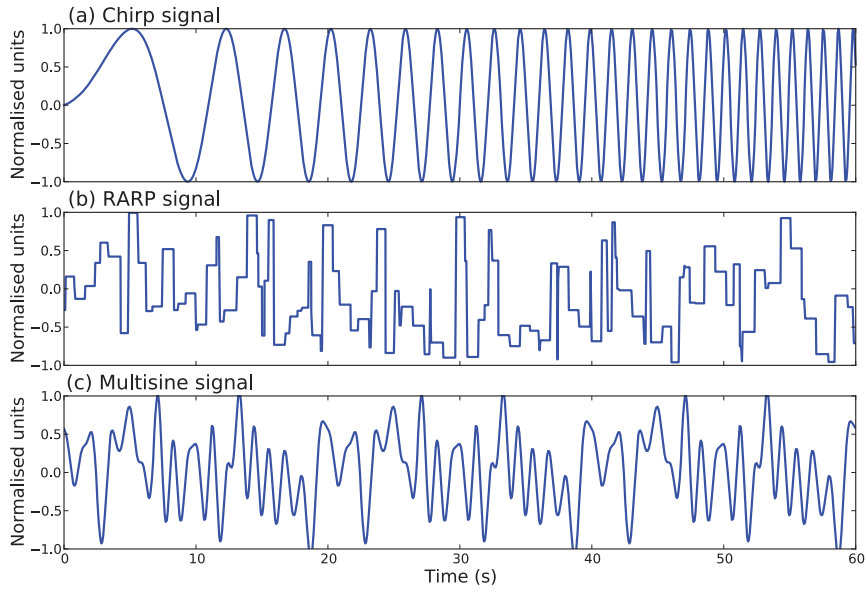

Fig. 3. Time series of potential identification signals.
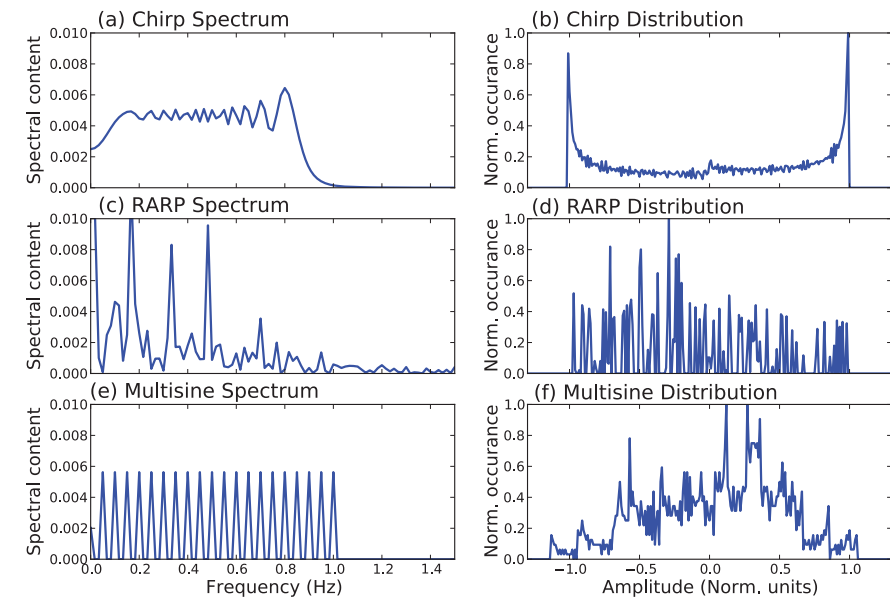

Fig. 4. Spectrum and amplitude distribution for signals in Fig. 3.

respective frequency and amplitude domain coverage. Each signal has different attributes regarding their spectral and amplitude properties, and also regarding the dependence of these properties on the signal length. Obviously, from a CFD computation standpoint, the shorter the simulation length, the better.

The chirp signal's (Fig. 3-(a)) frequency linearly sweeps a desired range during the evolution of the signal; therefore, its frequency content is distributed fairly evenly across that range, shown in Fig. 4-(a). The amplitude of the chirp signal is well bounded (Fig. 4-(b)), with free choice in setting the maximum amplitude, however, there is no free choice over the amplitude distribution.

The frequency content (Fig. 4-(c)) and amplitude distribution (Fig. 4-(d)) of the RARP vary due to the inherent randomness of the signal. However as the signal length increases, the amplitude distribution converges to an even coverage of the desired amplitude range and the frequency content converges to a distribution influenced by the maximum allowable random switching period (1s for the signal in Fig. 3-(b)). The amplitude distribution can be controlled by filtering the random numbers produced for the amplitude with a desired probability distribution function (e.g. Gaussian).

The multisine allows strong control over the spectral content (Fig. 4-(e)), with free choice for the amplitude of each harmonic. The amplitude distribution (Fig. 4-(f)), is determined 
by the amplitude and the phases of the harmonic components, which leads to control over the amplitude content in the multisine signals through phase optimisation techniques [19]. The multisine signal in Fig. 3 has a fundamental frequency of $0.05 \mathrm{~Hz}$ and equal amplitude for every harmonic up to $1 \mathrm{~Hz}$ with random phases.

Free surface elevation (FSE) and wave excitation force signals are constrained by underlying fluid dynamics (e.g. no square waves), therefore the possibility of utilising RARP or chirp signals is limited to excitation using an external (e.g. PTO) force input. It is not possible to create input FSE multisine signals with a flat broadbanded spectrum in the NWT due to limiting wave steepness, higher frequency waves have shorter wavelengths and therefore smaller maximum allowable amplitudes. For typical wave spectrums observed in the ocean, the spectral content for frequencies $(f)$ above the peak decay with a $f^{-5}$ tail [20]. However, external forces on the WEC from the mooring and PTO can contain high frequency components, which can be replicated by a direct force signal to the WEC in the NWT.

\section{CASE STUDY}

Here a case study is presented to illustrate the design and implementation of NWT experiments for the identification of WEC models. The data produced from the numerical wave tank experiments reported in this paper are then employed in [1] to determine suitable black-box hydrodynamic models for the device under test.

\section{A. Case Study Description}

The case study considers a two dimensional (2D) NWT, whereby the NWT is one cell thick and symmetry planes are imposed on the front and back faces of the domain. The 2D NWT is used to allow timely investigation of the optimal experiment design, before moving to the much more computationally complex 3D NWT for real WEC geometries.

The NWT is implemented using OpenFOAM, as detailed in [21]. The tank geometry is depicted in Fig. 6, having a 50m depth and walls located $100 \mathrm{~m}$ from the device, with wave creation/absorption implemented via the waves2FOAM package [22] utilising two $90 \mathrm{~m}$ long relaxation zones situated $10 \mathrm{~m}$ either side of the device. A 2D circular device geometry is simulated, which relates to the cross-section of a horizontally aligned cylinder of infinite length. The radius of the cylinder is $1 \mathrm{~m}$ with a density of half that of the water so that it rests $50 \%$ submerged. Fig. 5 shows a view of the mesh around the cylinder.

The cylinder's motion is constrained to heave, in all experiments, to allow the identified models in [1] to focus on this single degree of freedom for simplicity and clarity of presentation. Depending on the operational characteristics of the device, it may be desirable to identify models with multiple degrees of freedom (DoF). The NWT is well suited for this purpose, allowing the device motion to be constrained or actuated along any number of coupled modes of motion, which is very difficult to implement mechanically in a real wave tank. Although passing to multiple DoF will considerably increase the simulation time

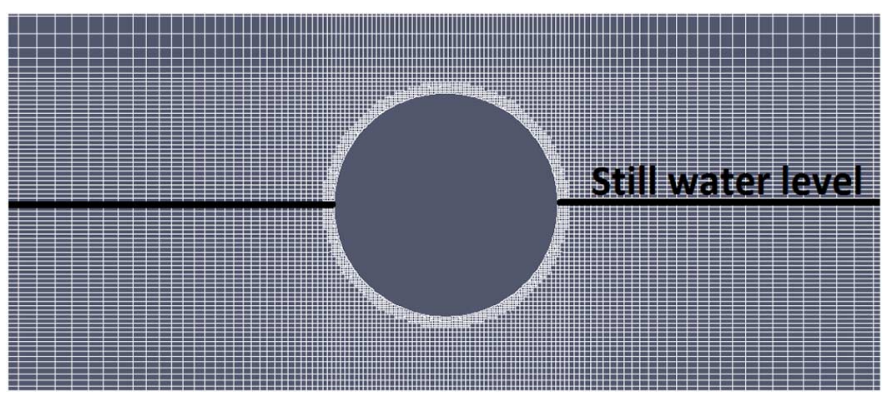

Fig. 5. Side view of the $2 \mathrm{D}$ geometry and the mesh used in the CFD simulation.

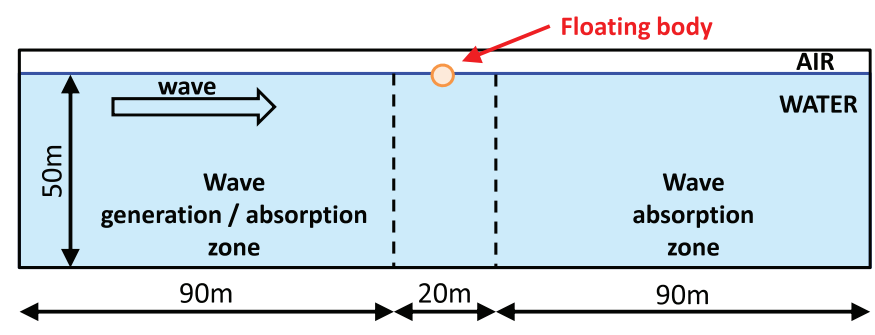

Fig. 6. Tank used for the case study experiments.

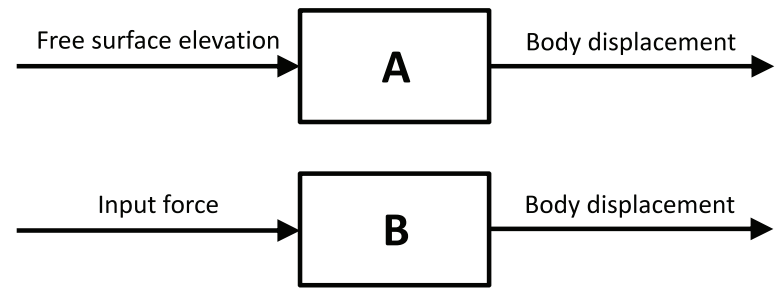

Fig. 7. Block diagram of the two different model types considered in the case study.

of the experiment, requiring all possible combinations of amplitude and frequency for each DoF to be excited, the ever increasing power and reducing cost of high performance computing allows well designed multiple DoF simulations to be feasible.

The identification of two different types of models is considered in this case study: FSE to body motion and input force to body motion, as outlined in Fig 7. Section IV-B details the wave excitation experiments used to provide identification data for the FSE to body motion model, while Section IV-C details the input force experiments for the input force to body motion models.

\section{B. Wave Excitation Experiments}

The wave excitation experiments are used to identify a model between the FSE and the device motion (Block A - Fig. 7). Two time series vectors are produced by these experiments for the model identification in [1], one containing the FSE, $\{\eta(k)\}$, and the other the body displacement, $\{y(k)\}$. A wave creation zone on one side of the device is used to create the input wave time series and an absorption zone on the leeward side of the device prevent these waves from being reflected back, as shown in Fig. 6.

The identified model considers as input, the FSE at the body's centre of mass, which can not be measured directly whilst the body occupies this space. Therefore, the experiment 


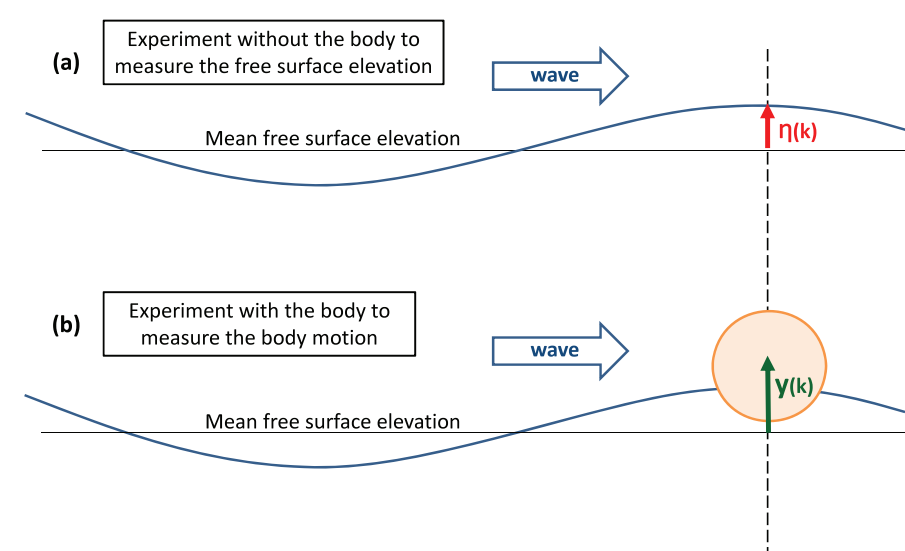

Fig. 8. Schematic of the procedure for the wave excitation experiments.
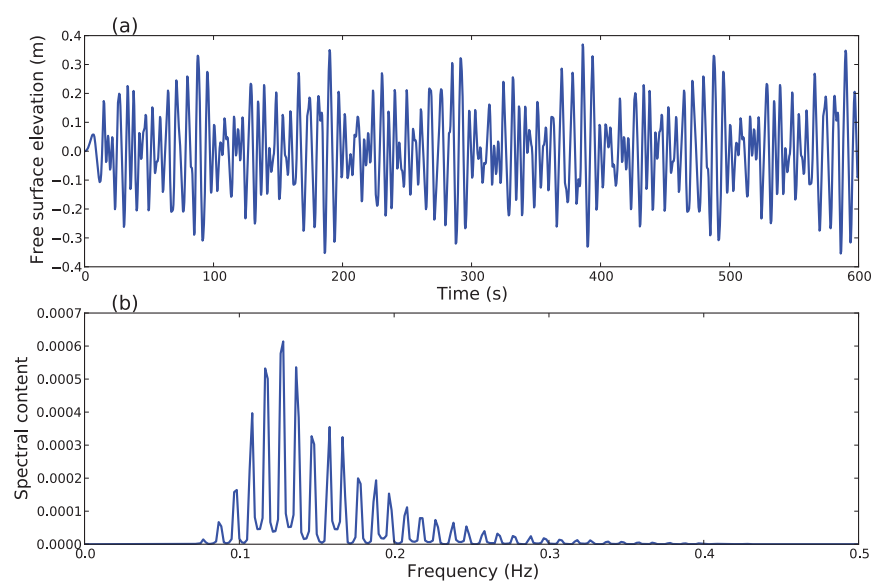

Fig. 9. (a) Input wave signal created on the free surface of the NWT, and (b) the corresponding frequency spectrum.

is performed in two stages; first, the input waves are created without the body in the tank, and the FSE measured at the desired position to obtain $\{\eta(k)\}$. Next, the same input waves are generated, but with the body in the tank, and the resulting body motion recorded to obtain $\{y(k)\}$. This procedure is illustrated in Fig 8.

The input wave signal is a 600s multisine signal, shown in Fig. 9-(a), consisting of 100 equally spaced frequencies from $0.005-0.995 \mathrm{~Hz}$ with randomly assigned phases and amplitudes determined by targeting a JONSWAP spectrum with a peak period of $8 \mathrm{~s}$, significant wave height $\left(H_{s}\right)$ of $0.6 \mathrm{~m}$ and a peakiness $(\gamma)$ value of 2. Fig. 9-(b) shows the frequency content of the input signal by plotting the Fourier transform of the FSE measured from the NWT experiment. The experiment is performed twice, using the same input wave spectrum but with different random phases for each frequency, to produce one data set for model training and a second for model validation in [1]. Fig. 10 shows a plot of both the FSE and resulting body motion from part of the wave excitation experiments.

To show the comparability of wave and input force excitations, in terms of amplitude of body motion, Fig. 11 shows the relative displacement (distinct from body position) of the device with respect to water surface. This will be seen to be commensurate with the relative displacement for the direct force tests, where no external waves are present.

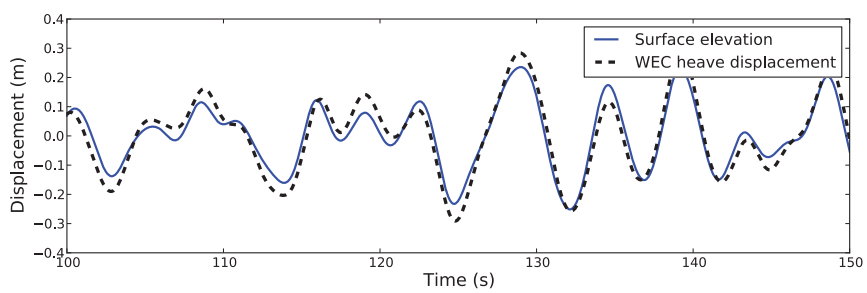

Fig. 10. Simulated free surface elevation, and corresponding body motion, measured from the NWT wave excitation experiments.

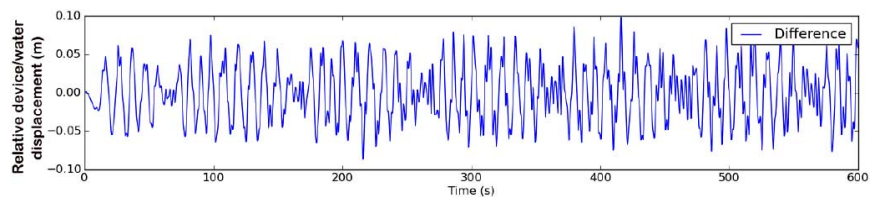

Fig. 11. Relative displacement of device with respect to water surface for wave excitation.

\section{Input Force Experiments}

The input force experiments are used to identify a model between an input force and the device motion (Block B - Fig. 7). Two time series vectors are produced by these experiments for the model identification in [1], one containing the input force, $\left\{f_{I N}(k)\right\}$, and the other the body displacement, $\{y(k)\}$. The input force experiments use wave absorption zones on both sides of the device to prevent waves radiated by the device from being reflected from the side walls and contaminating the results.

Unlike the input wave experiment, where the choice of input signal is constrained by the laws of fluid dynamics, the input force experiment allows total freedom over the choice of input signal to be applied to the system. It is important to ensure that the input signal covers the frequency range of interest and that input power is applied to the parts of the spectrum where the identified model is required to perform well. Fig. 12 depicts a block diagram of the input force type model for the wave energy case, where the input force is the sum of the wave excitation, PTO and mooring forces. Therefore, the input frequency range not only depends on the wave spectra in which the WEC is expected to operate, but also on the mooring and PTO loads, which may subject the device to high frequency inputs from mooring snap loads, maximum stroke end-stop collisions, latching control etc. The pragmatic approach, taken in this study, is to perform preliminary experiments (Section IV-C1) to ascertain some rough measurement of the resonant frequency and bandwidth of the device, informing the identification experiments (Section IV-C2) where their input power spectrum should be allocated.

1) Preliminary Experiments: Two simple preliminary experiments can be used to determine the frequency bandwidth of the device's natural and forced responses. Both preliminary experiments involve a very low total amount of kinetic energy in the device and the NWT fluid, which results in relatively fast CFD simulations. This allows the preliminary experiments to be quickly simulated before the identification experiments, to inform the design of the identification signals' frequency range. 


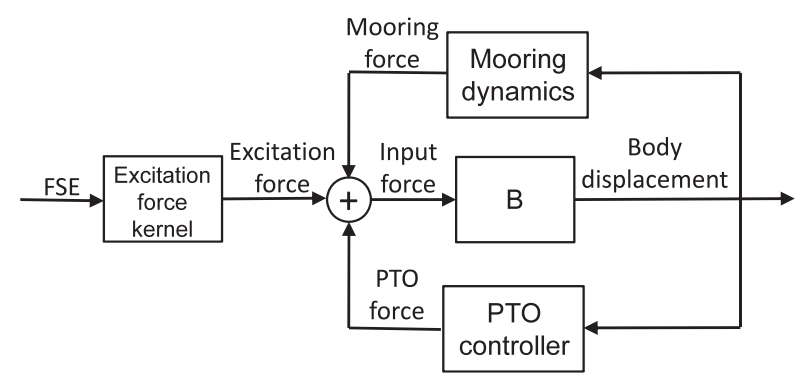

Fig. 12. Representation of the input force to a WEC model.
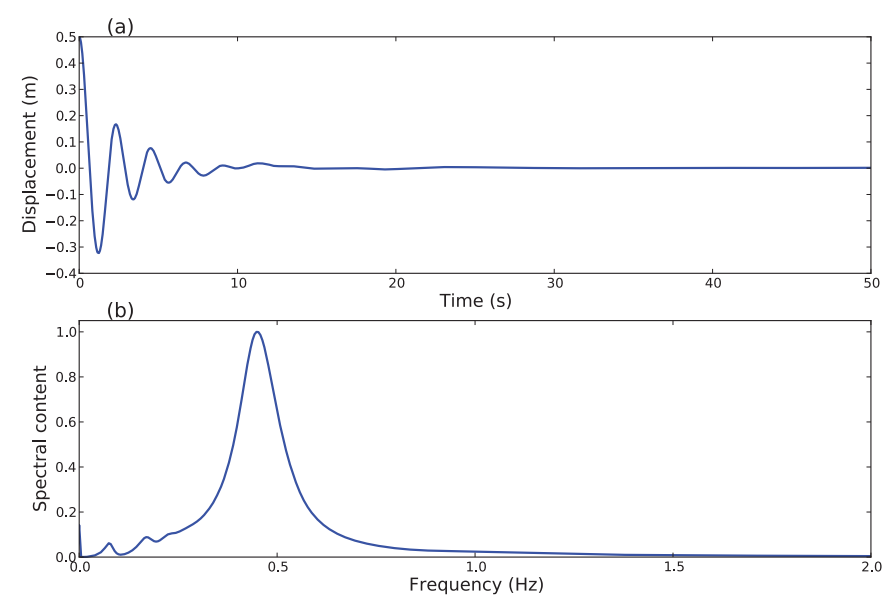

Fig. 13. Device free response in (a) time and (b) frequency domains.

The device's natural response is determined via a free decay experiment, whereby the device is initially displaced from its equilibrium position and allowed to freely oscillate back to rest. Fig. 13-(a) shows a free decay experiment performed on the case study set-up and Fig. 13-(b) displays the Fourier transform of the free decay and gives an indication of the device resonance and bandwidth (state dynamics).

The device's forced response is determined via a small amplitude chirp signal experiment, whereby a chirp signal is applied as a direct force on the device. Unlike the chirp signal used for the later identification experiments, which must cover a desired amplitude range, the chirp signal in the preliminary experiments is of very low amplitude allowing fast simulation times. The small amplitude chirp signal experiment therefore gives a frequency response identification of the total forced dynamics around the equilibrium position. This response is shown in Fig. 14.

Figs 13 and 14 give an indication of the system's bandwidth, where both figures show a resonant peak around $0.45 \mathrm{~Hz}$. Inspection of the frequency response informs the frequency range for which the identification experiments should be valid for. For this case study, the identification experiments will be designed to span the region of $0-1.5 \mathrm{~Hz}$.

2) Identification Experiments: Three different signal types are investigated for the input force experiments, a RARP signal, a multisine signal and a chirp signal input. Each signal is 600s in length and is applied as a direct vertical force acting on the device. The signals are designed to span the force amplitude

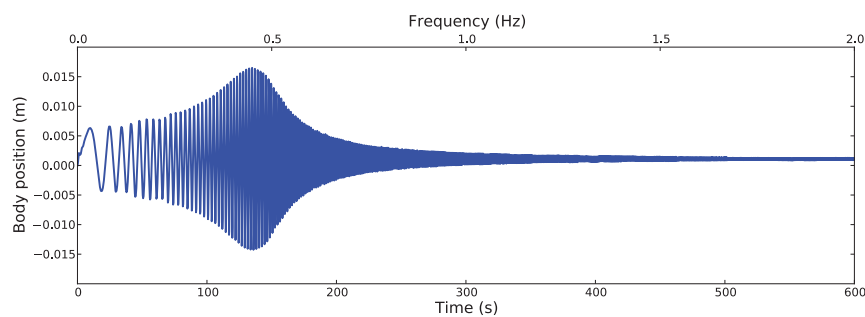

Fig. 14. Preliminary frequency response determination using a small amplitude chirp signal as input.
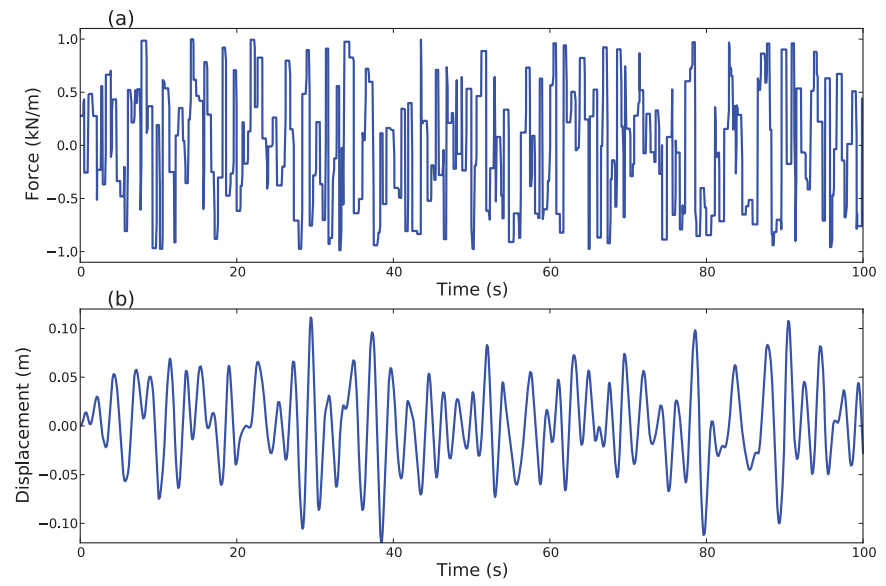

Fig. 15. A 100 second portion of the RARP signal used for identification, (a) input and (b) output.

range of $\pm 1 \mathrm{kN}$ per meter of cylinder length in the horizontal direction of the $2 \mathrm{D}$ symmetry planes $( \pm 1 \mathrm{kN} / \mathrm{m})$, and the frequency range of $0-1.5 \mathrm{~Hz}$. Two versions of each signal are generated: one to be used for model training and the second for model validation.

The RARP signal is designed with a maximum random switching period of $0.67 \mathrm{~s}(1.5 \mathrm{~Hz})$ and random force amplitudes with even probability across the $\pm 1 \mathrm{kN} / \mathrm{m}$ range. The signal is randomly generated twice under these constraints, with one signal being used for model training and the second for model validation in [1]. A sample of the input RARP force is plotted in Fig. 15-(a) and the resulting body motion in Fig. 15-(b).

The multisine signal, is designed with a flat frequency spectrum with a fundamental frequency of $1 / 600 \mathrm{~Hz}$ and all harmonics up to $1.5 \mathrm{~Hz}$. All frequency components are given equal amplitudes, to create a flat spectrum, and are assigned random phases. The amplitude of the frequency components is manually tuned until the time series distribution spans the range $\pm 1 \mathrm{kN} / \mathrm{m}$. The signal is generated twice using the same frequency spectrum but with different random phases, with one signal for model training and the second for model validation. The input force multisine signal is plotted in Fig. 16-(a) and the resulting body motion in Fig. 16-(b).

The chirp signal is designed to linearly sweep from 0 to $1.5 \mathrm{~Hz}$ with a maximum oscillation amplitude of $1 \mathrm{kN} / \mathrm{m}$. Fig. 17-(a) shows the body motion from the chirp signal used for model training. To generate a second signal for model validation, the chirp signal is reversed, linearly sweeping from 1.5 

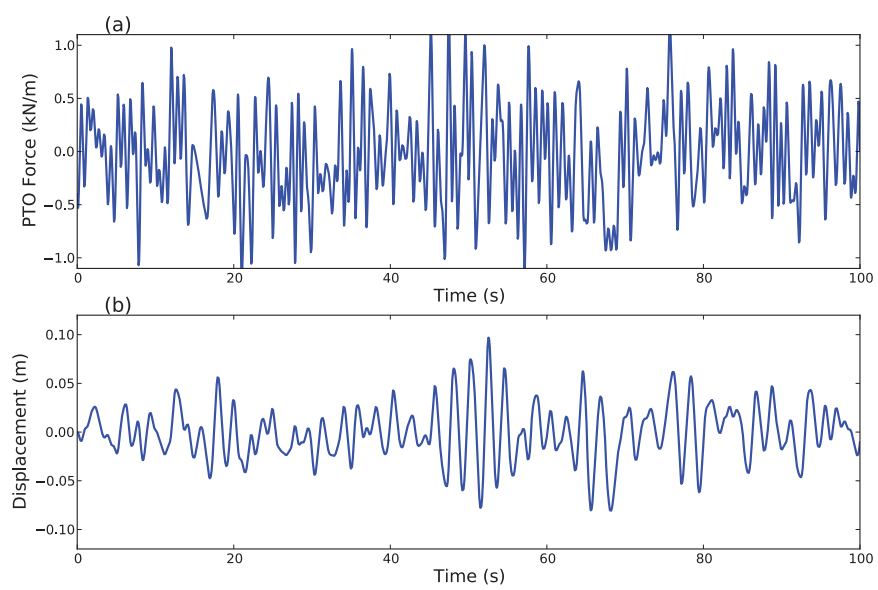

Fig. 16. A 100 second portion of the multisine signal used for identification, (a) input and (b) output.
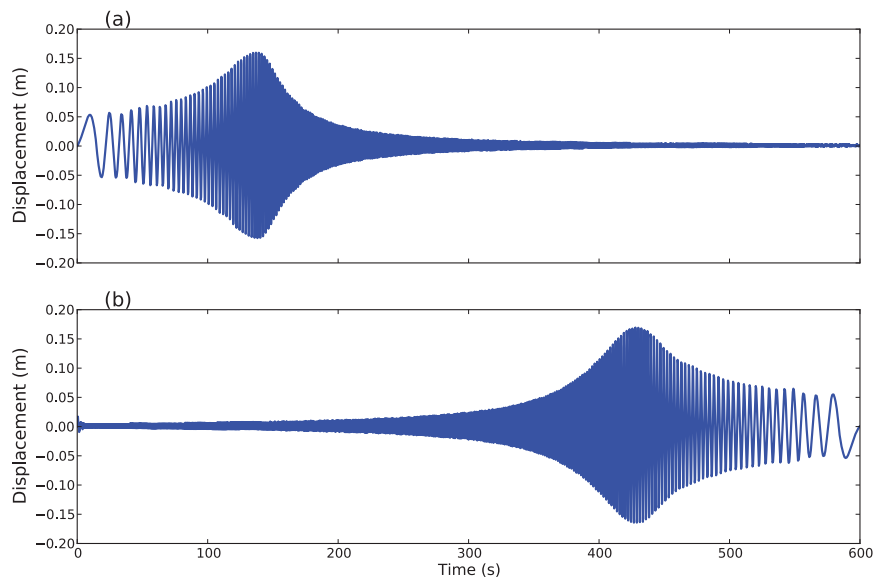

Fig. 17. Output of chirp signal experiment used for (a) model identification and (b) model validation.

to $0 \mathrm{~Hz}$. The resulting body motion from the reversed chirp signal used for model validation is shown in Fig. 17-(b).

\section{DISCUSSION}

Identifying WEC models from recorded NWT data requires that the data is representative of the conditions that the models will encounter. The design of the NWT experiments is therefore dependent on the required use of the model. The case study presented in Section IV-B considered a model to be used to simulate device motion in the presence of input waves. The frequency and amplitude range for these experiments is limited by the underlying fluid dynamics and limiting wave steepness (e.g. there are no square shaped waves on water), which are observed in the open ocean as well as the NWT under noraml WEC device power production conditions. The choice of input signals should be guided by the sea states the WEC is expected to operate in. The experiments can be designed using either specific individual sea spectrums, or by creating a generalised broadbanded spectrum spanning the entire set of expected spectrums likely to be encountered at a location. As example, the frequency content and amplitude distribution of the input FSE signal for the presented case study was designed to replicate a

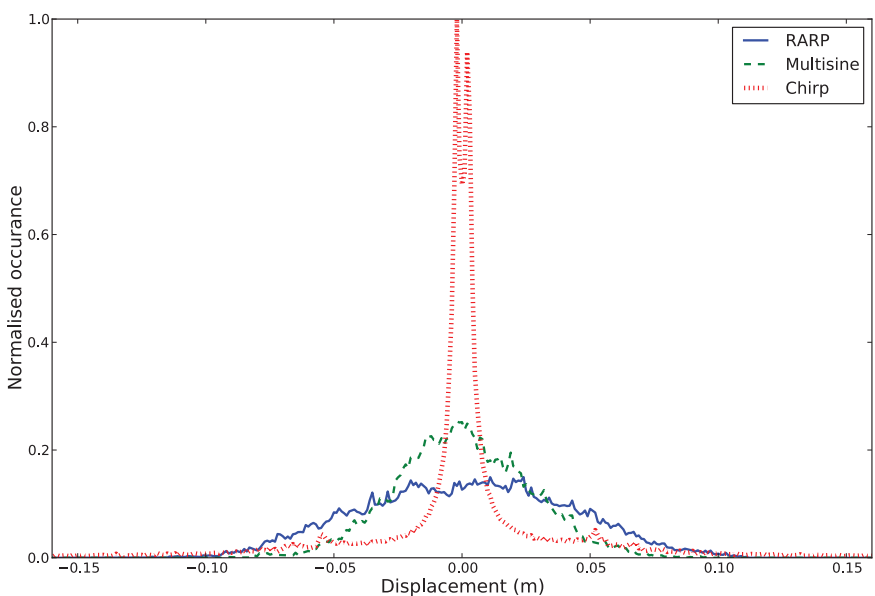

Fig. 18. Distribution density plot of the output device displacement excited by the different input signals.

commonly occurring sea state at the EMEC test site [23]. The tank depth of 50m was also chosen to correspond to the water depth at the EMEC site.

The input for the wave excitation model in Section IV-B was the FSE at the centre of the device. The use of such models is generally for predictive assessment of device performance using pre-measured wave data. In other situations, wave excitation models can be used for real-time control and take upstream measurements of the wave field as input. This type of scenario can be replicated in the NWT, by measuring the upstream FSE and the body motion in a single experiment, unlike the case in Section IV-B where it was necessary to measure the FSE and body motion in separate experiments. Another possible input variable to this type of model could be the pressure value probed at a location in the NWT, to replicate a pressure sensor mounted on the surface of the device or on the sea floor.

For nonlinear systems, which exhibit an input/output amplitude dependence, it is important for the signals to also span the amplitude range of interest. For the case in presented in Section IV-B the amplitude range was determined by the input sea state. For Section IV-C2, the input amplitude range investigated was 0 to $1 \mathrm{kN} / \mathrm{m}$, and three different signal types (chirp, RARP and multisine) were designed to span this range, as well as the specified frequency range ( 0 to $1.5 \mathrm{~Hz})$. The fidelity of the identified models in the companion paper [1], may be used an indicator of the efficacy of these three identification signals, both from validation and cross validation results. The identified models in [1] are nonlinear autoregressive with exogenous input (NARX) models, whereby the present output value is determined from the input as well as past output values. Therefore, the output amplitude range excited by the input signals is also important. The output amplitude distribution is plotted in Fig. 18, where it can be seen that the chirp signal excites the largest amplitude range but also spends the majority of its time around the equilibrium, due to the small response to the high frequencies in the tail of Fig 17. The multisine signals shows a more broadbanded output amplitude distribution and the RARP is seen to have the most evenly distributed output amplitude of the three signals. 
For the case study, the models are used for the heave motion of the floating body; therefore, the body is constrained to this single degree of freedom in the NWT. This is a nontrivial task in physical wave tanks requiring mechanical restraints which can alter the device dynamics and add friction to the system. It is also possible to perform multi-degree of freedom experiments in the NWT, with free choice over which modes of motion, if any, are to be constrained.

\section{CONCLUSION}

The field of system identification is successfully used for many applications and is demonstrated in this paper, and its companion [1], as a viable solution for obtaining WEC models. One difficulty, in using system identification for WEC modelling, is obtaining appropriate input/output data from open ocean or physical tank testing; however, the use of CFD based NWTs offers an avenue to overcome these difficulties and provide high fidelity device simulation to provide the data for model identification. The identification experiments for data generation must be well designed to ensure the data has good coverage of the expected operational conditions while making economical use of the test time.

\section{REFERENCES}

[1] S. Giorgi, J. Davidson, and J. Ringwood, "Identification of wave energy device models from numerical wave tank data-Part 2: Data-based model determination," IEEE Trans., to be published.

[2] L. Ljung, System Identification: Theory for the User, 2nd ed. Englewood Cliffs, NJ, USA: Prentice-Hall, 1999.

[3] Y. Nievergelt, "A tutorial history of least squares with applications to astronomy and geodesy," J. Comput. Appl. Math., vol. 121, no. 1, pp. 37-72, 2000.

[4] L. Ljung, "Perspectives on system identification," Annu. Rev. Control, vol. 34, no. 1, pp. 1-12, 2010.

[5] M. Bhinder, A. Babarit, L. Gentaz, and P. Ferrant, "Assessment of viscous damping via 3D-CFD modelling of a floating wave energy device," in Proc. Eur. Wave Tidal Energy Conf. (EWTEC), 2011.

[6] L. Bonfiglio, S. Brizzolara, and C. Chryssostomidis, "Added mass and damping of oscillating bodies: A fully viscous numerical approach," in Recent Advances in Fluid Mechanics, Heat \& Mass Transfer and Biology. Cambridge, USA: WSEAS Press, 2011.

[7] J. Davidson, S. Giorgi, and J. Ringwood, "Linear parametric hydrodynamic models based on numerical wave tank experiments," in Proc. Eur. Wave Tidal Energy Conf. (EWTEC), Aalborg, Denmark, 2013.

[8] J. Davidson, S. Giorgi, and J. V. Ringwood, "Linear parametric models for wave energy converters identified from numerical wave tank experiments," Ocean Eng., vol. 103, pp. 31-39, 2015.

[9] J. A. Armesto, R. Guanche, A. Iturrioz, C. Vidal, and I. J. Losada, "Identification of state-space coefficients for oscillating water columns using temporal series," Ocean Eng., vol. 79, pp. 43-49, 2014.

[10] J. Davidson, S. Giorgi, and J. Ringwood, "Numerical wave tank identification of nonlinear discrete-time hydrodynamic models," in Proc. 1 st Int. Conf. Renew. Energies Offshore, Lisbon, Portugal, 2014, pp. 279-286.

[11] S. Giorgi, J. Davidson, and J. V. Ringwood, "Identification of nonlinear excitation force kernals using numerical wave tank experiments," in Proc. 11th Eur. Wave Tidal Energy Conf. (EWTEC), 2015, pp. 09C11109C1110.

[12] J. V. Ringwood, J. Davidson, and S. Giorgi, "Optimising numerical wave tanks tests for the parametric identification of wave energy device models," in Proc. 34th Int. Conf. Ocean Offshore Arctic Eng. (OMAE'15), 2015, pp. OMAE2015-41 529-1-OMAE2015-41 529-10.

[13] K. Tanizawa, "The state of the art on numerical wave tank," in Proc. 4th Osaka Colloquium Seakeeping Perform. Ships, 2000, pp. 95-114.
[14] I. Currie, Fundamental Mechanics of Fluids. New York, NY, USA: McGraw-Hill, 1974.

[15] A. Iturrioz, R. Guanche, J. Lara, C. Vidal, and I. Losada, "Validation of openfoam $\AA$ for oscillating water column three-dimensional modeling," Ocean Eng., vol. 107, pp. 222-236, 2015.

[16] Y. Wei, A. Rafiee, A. Henry, and F. Dias, "Wave interaction with an oscillating wave surge converter-Part I: Viscous effects," Ocean Eng., vol. 104, pp. 185-203, 2015.

[17] P. Schmitt and B. Elsaesser, "On the use of openfoam to model oscillating wave surge converters," Ocean Eng., vol. 108, pp. 98-104, 2015.

[18] V. Mishra, S. Beatty, B. Buckham, P. Oshkai, and C. Crawford, "Application of an arbitrary mesh interface for CFD simulation of an oscillating wave energy converter," in Proc. 11th Eur. Wave Tidal Energy Conf., 2015, pp. 07B141-07B1410.

[19] R. Pintelon and J. Schoukens, System Identification-A Frequency Domain Approach, 2nd ed. Hoboken, NJ, USA: Wiley, 2012.

[20] M. J. Tucker and E. G. Pitt, Waves in Ocean Engineering. Amsterdam, The Netherlands: Elsevier, 2001, vol. 5.

[21] J. Davidson, M. Cathelain, L. Guillemet, T. Le Huec, and J. Ringwood, "Implementation of an openfoam numerical wave tank for wave energy experiments," in Proc. Eur. Wave Tidal Energy Conf. (EWTEC), 2015, pp. 09B111-09B1110.

[22] N. G. Jacobsen, D. R. Fuhrman, and J. Fredsøe, "A wave generation toolbox for the open-source CFD library: Openfoam®," Int. J. Numer. Methods Fluids, vol. 70, pp. 1073-1088, 2012.

[23] A. Babarit, J. Hals, M. Muliawan, A. Kurniawan, T. Moan, and J. Krokstad, "Numerical benchmarking study of a selection of wave energy converters," Renew. Energy, vol. 41, pp. 44-63, 2012.

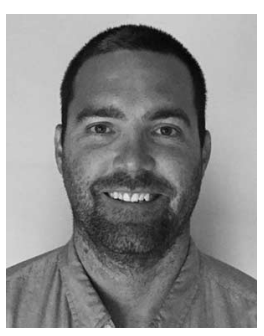

Josh Davidson received the Bachelor's degree (first class Hons.) in physical sciences from James Cook University, Townsville City Qld., Australia, in 2006, and the Ph.D. degree in energy harvesting for marine-based sensors from James Cook University, Townsville City, Qld., Australia. He was co-funded by the Commonwealth Scientific and Industrial Research Organisation's (CSIRO) Energy Technology Division, working with their 'Energy Harvesting Team'. Currently, he works with the Centre for Ocean Energy Research, Maynooth University, Maynooth, Ireland, where his research explores nonlinear modelling for wave energy converters through numerical wave tank experiments.

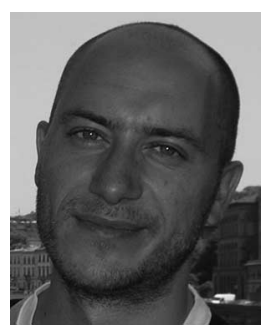

Simone Giorgi received the Master's degree (first class Hons.) in electronics at the Universita degli Studi di Ferrara (UNIFE), Ferrara, Italy, in 2002, and the Master's degree (first class Hons.) in renewable energy systems from Maynooth University, Maynooth, Ireland. He is currently pursuing the Ph.D. degree at the Centre for Ocean Energy Research, Maynooth University. His research interests include nonlinear parametric modelling and control for wave energy devices using numerical tank testing.

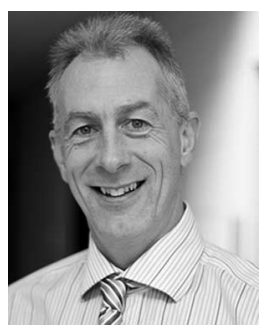

John V. Ringwood (SM'97) received the Diploma degree in electrical engineering from Dublin Institute of Technology, Dublin, Ireland, and the Ph.D. degree in control systems from Strathclyde University, Glasgow, U.K., in 1981 and 1985, respectively. He is currently a Professor of Electronic Engineering with the National University of Ireland (NUI), Dublin, Ireland, and the Director of the Centre for Ocean Energy Research (COER), NUI Maynooth, Maynooth, Ireland. He was the Head of the Electronic Engineering Department, NUI Maynooth from 2000 to 2005, developing the Department from a greenfield site. His research interests include time series modelling, wave energy, and biomedical engineering. $\mathrm{He}$ is a Chartered Engineer. He is a Fellow of the Institution of Engineers of Ireland. 